\title{
Healthcare providers' attitudes and perceptions in infection diagnosis and antibiotic prescribing in public health institutions in Lesotho: a cross sectional survey
}

\author{
*Adorka $\mathrm{M}^{1}$, Dikokole $\mathrm{M}^{2}$, Mitonga $\mathrm{KH}^{3}$, Allen $\mathrm{K}^{4}$
}

1. University of Namibia, Faculty of Health Sciences, School of Medicine, School of Pharmacy, Windhoek, Namibia.

2. National University of Lesotho, Faculty of Social Sciences, Department of Statistics, Roma, Lesotho.

3. University of Namibia, Faculty of Health Sciences, School of Medicine, Department of Community Medicine, Windhoek, Namibia.

4. Medical and Pharmaceutical Biotechnology Unit, Research Center in Technology and Design Assistance of Jalisco State (CIATEJ,A.C) Guadalajara, Mexico.

\begin{abstract}
Background: The decision to prescribe antibiotics and the selection of an appropriate antibiotic are important in the treatment of infectious diseases. As any human decision, it can be influenced by individual attitudes and perceptions.

Objectives: To assess the attitudes and perceptions of healthcare providers regarding antibiotic prescribing and the use of laboratory results in infection diagnosis.

Methods: A cross sectional survey in five selected Health Service Areas (HSAs) in the Southern Africa country of Lesotho. The questionnaires were self-administered to 67 healthcare providers in public health institutions within the selected HSAs. Data were analyzed using Fisher's exact test or the McNemar Test for dependent proportions.

Results: 51 surveys were returned ( 39 medical doctors, 12 nurses). Respondents typically practiced in urban settings, worked with both inpatients and outpatients, had over 10 years experience, and attended to at least 26 patients per day. We identify several inappropriate practices related to the use of on-site microbiology laboratories. For example, only $17 \%$ always send a sample for microscopic identification prior to prescribing antibiotics and only $32 \%$ always send a sample for culture sensitivity tests. Delays in obtaining laboratory results and high patient workloads were cited as reasons for under-utilization of laboratory facilities. Nearly all respondents recognize the need for guidelines and further training in antibiotic prescribing. Conclusions: Healthcare providers demonstrated attitudes and perceptions in antibiotic prescribing or use of laboratory derived information in infection diagnosis that could have negative impacts on antibiotic prescribing.

Key words: Healthcare providers, Lesotho, antibiotic prescribing, laboratory derived information

African Health Sciences 2013; 13(2): 344 - 350 http://dx.doi.org/10.4314/ahs.v13i2.21
\end{abstract}

\section{Introduction}

Antimicrobial overuse, misuse and even under use are elements of inappropriate prescribing and are key drivers of the development bacterial pathogen resistance ${ }^{1,2,3}$. Antibiotic resistance was first identified shortly after widespread introduction of the drugs during World War II ${ }^{4,5}$. In respiratory tract infections, for example, inappropriate prescribing of antibiotics contributes to the development of drug resistance of the most common bacterial pathogens causing the infection ${ }^{2}$. Antibiotic-resistant microbes are common in Africa, and many studies of antibiotic

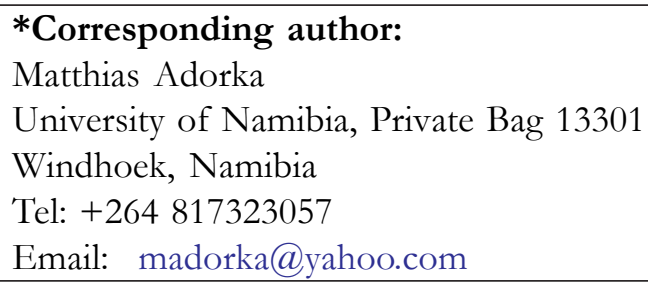

resistance have been conducted in Lesotho's neighbor South Africa (reviewed in Crowther-Gibson, et al. ${ }^{6}$ ). Most healthcare providers are aware of the consequences of inappropriate prescribing and use of antibiotics ${ }^{3}$, and stewardship programs can successfully combat the misapplication of antibiotics ${ }^{7,8}$. Nevertheless, healthcare providers continue to prescribe antibiotics inappropriately, creating a problem that needs urgent attention.

A number of studies attribute inappropriate prescribing to factors related to health care providers' attitudes and perceptions of antibiotic prescribing. These factors include, for example: patients' expectations of antibiotic treatments having effect on physicians' prescribing behavior; healthcare providers' decision to prescribe antibiotics to be on the safer side when there is diagnostic uncertainty; 
lack of healthcare providers' knowledge regarding optimal diagnostic approaches; insufficient training of healthcare providers in infectious diseases and antibiotic treatment; and difficulty of selecting antiinfective drugs considered most appropriate in the empiric treatment of infections ${ }^{3,9,10}$. These factors may not, however, answer all questions pertaining to causes of inappropriate antibiotic prescribing since prescribing patterns differ in different clinical environments.

To be appropriately used with good chances of effectiveness, antibiotics need to be prescribed against infections of pathogens for which they are indicated $^{1,10}$. As a fundamental principle in the judicious prescribing of antibiotics, bacterial aetiologies of infections need to be established prior to antibiotic prescribing. This can be challenging in situations where presenting signs and symptoms of the diagnosed case may not indicate the presence of bacterial pathogens as absolute aetiologies of the infection. However, it is possible to accurately diagnose bacterial causes of infections if proper diagnostic workups are carried out. Such workups ordinarily include physical examinations and considerations of additional predictors of bacterial infections, e.g., illness lasting for seven or more days in the case acute rhinosinusitis and acute bronchitis may have to be supported by laboratory investigations ${ }^{11}$.

Healthcare providers' attitudes and perceptions towards the use of antibiotics in treating a presenting clinical condition influence whether they prescribe antibiotics or use laboratory derived information in identifying causative agents of infections. In events where such decisions are taken for the wrong reasons, the drugs are more likely to be prescribed inappropriately. This said, it is evident that investigating the extent to which health care provider attitudes affect decision making in antibiotic prescribing or the use of laboratory provided information in infection diagnosis would provide a valuable means of identifying fundamental causes of inappropriate prescribing. In many developing countries, unavailability of functional clinical microbiology laboratories or inefficiencies in the use of such facilities limit the use of laboratory provided information in infection diagnosis and appropriate selection of antibiotics ${ }^{12}$. Functional microbiology laboratories are available in all public hospitals in Lesotho. Therefore, the inefficient use rather than the unavailability of the facilities in the country's hospitals is more important in the non-use of laboratory provided information in infection diagnosis and treatment.

In Lesotho, antibiotics are prescribed by both doctors and nurses mostly in the empiric treatment of infections. In the absence of antibiograms and comprehensive antibiotic prescribing guidelines, decisions to prescribe antibiotics are based solely on opinions of healthcare providers. The researchers' search of the literature revealed no records of studies investigating factors that affect decisions of healthcare providers to prescribe antibiotics in Lesotho. It is therefore relevant to conduct this study in this southern African state for purposes of providing baseline information in formulating policies on appropriate prescribing of antibiotics.

The objective of the study was to assess attitudes and perceptions of healthcare providers in selected public health institutions in Lesotho regarding antibiotic prescribing and the use of laboratory provided information in infection diagnosis and the selection of antibiotics.

\section{Methods}

A cross-sectional study was conducted in June-July 2009 at five Health Services Areas (HSAs) in the southern African state of Lesotho. Study site selection was done on the basis of bed sizes and ownership of hospitals serving HSAs. Total bed capacities of hospitals within the five selected HSAs constituted nearly $50 \%$ of the total bed capacity of all hospitals in the country.

All doctors and primary healthcare nurses who prescribe antibiotics and also practice in public health institutions within HSAs were considered eligible for participation in the study. Of a total of 74 eligible health care providers identified, 67 agreed to participate and 51 returned the survey. The questionnaires were distributed directly to the respondents by the principal researcher. Completed questionnaires were collected from respondents either directly or from pre-arranged points where they were returned.

The questionnaire was semi-structured with mainly close-ended questions and a few open-ended questions. The order of questions largely followed the pattern provided by Aldrigde and Levine ${ }^{13}$, but the content was developed specifically for this study.

The survey was piloted in one hospital and one HSA in Lesotho with more than 10 medical doctors and nurses who prescribe antibiotics. The questionnaire was altered based on suggestions prior 
to full administration. Survey items asked the following: i) demographic data of respondents; ii) degrees to which patient and healthcare provider related factors influence care providers' decisions to prescribe antibiotics; iii) healthcare provider habits in prescribing antibiotics in outpatient and inpatient settings; iv) attitudes and perceptions of healthcare providers towards the use of laboratory generated information on characteristics of bacterial pathogens in infection diagnosis; v) opinions regarding the need for guidelines and further training in antibiotic prescribing.

\section{Statistical analysis}

Proportions were used to summarize the baseline characteristics of the respondents as data consist of categorical variables. In addition, associations between respondents' baseline characteristics and their attitudes and perceptions in infection diagnosis and antibiotic prescribing were summarized using contingency tables. Most survey items had three or more categories, and we dichotomized the categories as the strongest response (e.g. Always) vs. all others (e.g. Sometimes + Not at all) so that they could be analyzed as $2 \times 2$ tables. For comparing across groups (Doctors vs. Nurses), we used the Fisher Exact Test because expected cell frequencies were less than 5 . For most survey items, the response frequencies for Doctors and Nurses were not significantly different and we combined the results. For comparing within groups across survey items (e.g. Question 1 vs. Question 2), we used the McNemar Test for dependent proportions. In Tables 2 - 4, when two rows have the same letter, they are not significantly different. For all statistical tests, a value of $p<0.05$ was considered significant. All calculations were performed in the $\mathrm{R}$ software program, version 2.13.0.

\section{Ethical permission}

Ethical approval for this study was obtained from the Ethics Committee of the Lesotho Ministry of Health and the Research Ethics Committee of NorthWest University of South Africa (Permission Number 06K17), where the principal researcher compiled the report. Participating hospitals also granted permission to conduct the study.

\section{Results \\ Descriptive profile of the respondents}

The questionnaires were completed and returned by the healthcare providers in the selected HSAs at the overall response rate of $76 \%$ (51 of 67). The baseline characteristics of these health professionals are presented in table 1. Most of the respondents were Medical doctors $(77 \%)$ and practiced in an urban area $(84 \%)$. A majority of respondents (59\%) worked only in government health institutions, whereas respondents from only Christian Health Association (CHAL) constituted $29 \%$ and a small number $(16 \%)$ worked in both government and CHAL hospitals. Over half (59\%) of the respondents attended to patients in both outpatient and inpatient departments. Very few (4\%) reported attending to patients only in inpatient departments. Most (61\%) of the respondents had over 10 years of working experience, and a majority (65\%) reported that they see and treat between 26 and 100 patients per day.

Table 1: Baseline characteristics of healthcare providers who completed and returned the questionnaires $(n=51)$

\begin{tabular}{|c|c|c|}
\hline Characteristics & Categories & $n(\%)$ \\
\hline \multirow[t]{7}{*}{ Qualification } & Physician specialists & $11(22)$ \\
\hline & Surgical consultants & $3(6)$ \\
\hline & General practitioners & $25(49)$ \\
\hline & l: Medical Doctors & $39(76)$ \\
\hline & Nurse clinicians & $8(16)$ \\
\hline & Registered nurses & $2(4)$ \\
\hline & Nursing assistants & $2(4)$ \\
\hline \multicolumn{2}{|c|}{ Subtotal: Nurses } & $12(24)$ \\
\hline \multirow[t]{2}{*}{ Practice Location } & Urban & $41(80)$ \\
\hline & Rural & $10(20)$ \\
\hline \multirow[t]{2}{*}{ Practice Type } & Only Government of Lesotho (GOL) & $32(63)$ \\
\hline & Only Christian Hospital Association of Lesotho (CHAL) & $11(22)$ \\
\hline
\end{tabular}


Continuation of table 1

\begin{tabular}{llr}
\hline Characteristics & Categories & $\mathbf{n ~ ( \% )}$ \\
\hline \multirow{3}{*}{ Patient Type } & Both GOL \& CHAL & $8(16)$ \\
& Outpatient & $19(37)$ \\
& Inpatient & $1(2)$ \\
Work experience & Both outpatient \& inpatient & $32(63)$ \\
& Up to 5 years & $8(16)$ \\
& 6-10 years & $13(25)$ \\
Workload & Over 10 years & $30(59)$ \\
& 1 -25 patients/day & $11(22)$ \\
& 26 - 100 patients/day & $30(59)$ \\
& Over 100 patients/day & $8(16)$ \\
& No response & $2(4)$ \\
\hline
\end{tabular}

Table 2 shows the survey results to the question: "To what degree does any of the following factors influence your decision to prescribe antibiotics?" The questions are ordered by the percent of responses "Major degree". Prescribers reported that they are most influenced by the clinical conditions of the patient and are least influenced by patient requests or expectations $(73 \%)$. Nurses said that they are significantly more influenced by patient requests $(42 \%)$ and expectations $(33 \%)$ than doctors $(3 \%$ on each). For doctors, patient requests and expectations are significantly less influential than other factors $(p<0.05$, rows with the letter $\mathrm{D})$.

Table 2: Factors that influence the decision to prescribe antibiotics

\begin{tabular}{|c|c|c|c|c|c|c|}
\hline Factor & No response & Not at all & Minor degree & Major degree & $(\%)$ & \\
\hline \multicolumn{7}{|c|}{ Clinical condition of patient, i.e. biomedical factors } \\
\hline & 3 & 4 & 9 & 36 & $73 \pm 6$ & A \\
\hline \multicolumn{7}{|c|}{ Past experience with this type of clinical condition } \\
\hline & 3 & 5 & 15 & 27 & $55 \pm 7$ & $\mathrm{~B}$ \\
\hline \multicolumn{7}{|c|}{ Quest to prevent an infection even if I rule out the presence of bacterial infection } \\
\hline & 5 & 7 & 15 & 24 & $51 \pm 7$ & $\mathrm{~B} \quad \mathrm{C}$ \\
\hline \multicolumn{7}{|c|}{ Quest to eliminate an underlying infection of suspect in cases of unclear diagnosis } \\
\hline & 3 & 2 & 25 & 21 & $43 \pm 7$ & $\mathrm{C}$ \\
\hline \multicolumn{7}{|c|}{ Quest to satisfy patients' request for an antibiotic } \\
\hline Nurses & 0 & 4 & 3 & 5 & $\begin{array}{l}42 \pm 14 \\
*\end{array}$ & \\
\hline Doctors & 1 & 34 & 3 & 1 & $3 \pm 3$ & $\mathrm{D}$ \\
\hline \multicolumn{7}{|c|}{$\begin{array}{l}\text { Quest to satisfy patients' expectations regarding what treatment they think they } \\
\text { should be given for their presenting ailment }\end{array}$} \\
\hline Nurses & 0 & 6 & 2 & 4 & $\begin{array}{l}33 \pm 14 \\
*\end{array}$ & \\
\hline Doctors & 1 & 37 & $\overline{0}$ & 1 & $3 \pm 3$ & $\bar{D}$ \\
\hline
\end{tabular}

$(\%)$ is the percent of responses Major degree (excluding No response) \pm SEM

$*$ Nurses and Doctors significantly different $(p<0.05)$ by Fisher Exact Test (Major vs. Minor + Not at all). Rows with the same letter are not significantly different by the McNemar Test (Major vs. Minor + Not at all). For example, the first row (A) is significantly different from all other rows (B, C, D). When the Fisher Exact Test was significant, we excluded Nurses from the McNemar Test calculation. 
Table 3 shows the results to the question "If you prescribe in an out-patient setting, how often would you do what the following statements imply?" A majority of respondents reported that they always use clinical findings (74\%) and presenting signs and symptoms $(60 \%)$ when prescribing antibiotics. However, only $25 \%$ stated that they always await results of laboratory investigations and $51 \%$ sometimes prescribe antibiotics even if unsure of the diagnosis.

Table 3: Prescribers' practices regarding antibiotics prescribing among inpatients

\begin{tabular}{|c|c|c|c|c|c|c|}
\hline Behavior & No response & Never & Sometimes & Always & $(\%)$ & \\
\hline \multicolumn{7}{|c|}{$\begin{array}{l}\text { Only after I have examined a patient and my clinical findings positively establish } \\
\text { the presence of an infection }\end{array}$} \\
\hline & 1 & 0 & 13 & 37 & $74 \pm 6$ & A \\
\hline \multicolumn{7}{|c|}{$\begin{array}{l}\text { When the patient's presenting signs and symptoms make me suspect the presence } \\
\text { of an infection }\end{array}$} \\
\hline & 1 & 0 & 20 & 30 & $60 \pm 7$ & A \\
\hline \multicolumn{7}{|c|}{ Only after laboratory investigations have established the presence of infection } \\
\hline & 15 & 6 & 21 & 9 & $25 \pm 7$ & $\mathrm{~B}$ \\
\hline \multicolumn{7}{|c|}{ Sometimes even if I am not sure of my diagnosis } \\
\hline & 2 & 23 & 25 & 1 & $2 \pm 2$ & $\mathrm{C}$ \\
\hline
\end{tabular}

$(\%)$ is the percent of responses Always (excluding No response) \pm SEM. Rows with the same letter are not significantly different by the McNemar test (Always vs. Sometimes + Never). For example, the first two rows (A) are not significantly different from each other but are significantly different from the bottom two rows (B, C).

Table 4 shows responses to the question: "If you prescribe in an in-patient setting where facilities exist for you to perform culture sensitivity tests, what do you normally do in the course prescribing of an antibiotic?" Most respondents (79\%) indicated that they replace an initial prescription with another antibiotic sensitive to the microorganisms $(p<0.05$ compared to all other rows). However, only $32 \%$ said that they send a specimen for culture sensitivity testing and $17 \%$ said that they send a specimen for rapid microscopic identification and gram stain properties prior to beginning antibiotic treatment. Unavailability of antibiotic stocks at the hospital pharmacy is a common problem, with $53 \%$ of respondents indicating that it affects to a major degree their ability to treat infections. When the first choice antibiotic is not available, prescribers either direct patients to buy the first choice from a retail pharmacy $(61 \%)$ or, more commonly, prescribe a second choice which is available $(93 \%)$.

Laboratory tests are under-used due to the delay in receiving results: $68 \%$ of doctors said that results either take more than 8 hours or are rarely received before the prescription is made. They also cited their high patient load as a common reason for not requesting laboratory confirmation prior to prescribing. Providers indicated a willingness to improve antibiotic prescribing. Nearly all respondents believe guidelines (94\%) and refresher courses (88\%) would be helpful.

Table 4: Prescribers' practices regarding use of appropriate antibiotics

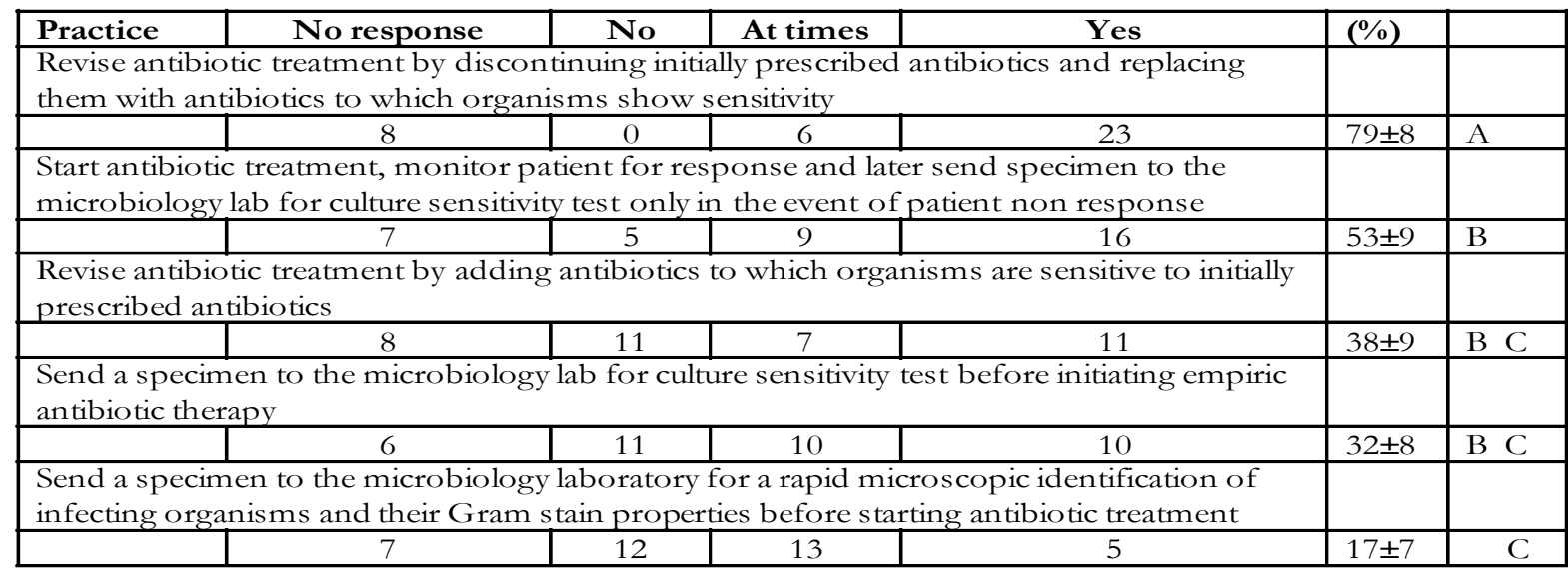




\section{Table 4 footnote:}

$(\%)$ is the percent of responses Yes (excluding No response) \pm SEM

Rows with the same letter are not significantly different by the McNemar test (Yes vs. At times + No). For example, the bottom row (C) is not significantly different from the two rows immediately above it (also C).

\section{Discussion}

Our results identified patient and healthcare provider related factors that influence health service providers to prescribe antibiotics inappropriately. There is no consensus on the role of patient requests and expectations in antibiotic prescribing. For example, parents' expectation for antibiotics to be used in treating pediatric cold was a minor factor in antibiotic over-prescribing in Korea ${ }^{14}$. Gaur and English also assert those patients' expectations for antibiotic treatments may not be a factor contributing to inappropriate antibiotic prescribing in developing as compared with developed countries ${ }^{10}$. However, other evidence suggests that patient demand has a strong influence on doctors' prescribing decisions ${ }^{15,16}$. In our survey, only $3 \%$ of doctors said that they prescribed antibiotics to satisfy patients' requests or expectations. On the other hand, $33-42 \%$ of nurses reported that they were influenced by patients' requests and expectations $(p<0.05$ compared to doctors).

We identified several other behaviors that are not optimal. For example, 51\% stated that they are influenced to a major degree to prescribe antibiotics even if an infection has been ruled out, and $43 \%$ are influenced to a major degree to prescribe antibiotics even if the diagnosis is unclear (table 2). Unavailability of the desired antibiotic in the hospital pharmacy is also an important influence (53\% to a major degree). Providers often recommend a second choice $(93 \%)$ in this case.

Factors limiting healthcare providers' use of laboratory provided information in infection diagnosis

Over one-third (40\%) of respondents with microbiology laboratory facilities at their practice sites reported that they fail to request microscopic identification of bacterial pathogens to aid their choices of antibiotics in treating infections, and another $43 \%$ request microscopic identification only at times (table 4). Only 25\% said that they always await laboratory results before prescribing (table 3), similar to a study in Nepal where only $20 \%$ of antibiotic prescriptions were preceded by a culture test ${ }^{17}$. The results suggest a significant percentage of antibiotics being prescribed for infections not adequately diagnosed by laboratory assisted information. It also suggests the high likelihood of inappropriate choices of antibiotics being made for prescribing. Respondent workloads in terms of number of patients seen and treated daily (table 1), as well as long time lags between requests for laboratory tests and the provision of results (68\% greater than 8 hours or not at all), are the major reasons for the high rate of non-use of laboratory facilities.

\section{Study limitations and strengths}

The sample size of respondents to questionnaires is small. Coupled with some respondents' unwillingness to respond to certain questions, this compromised the statistical significance of some results. We analyzed combinations of variables not presented in the tables (e.g. work experience or patient workload vs. antibiotic prescription attitudes), but the number of statistically significant results was not greater than would be expected by chance at thealpha $=0.05$ level. However, based on the high response rate and the fact that the investigation targeted all healthcare providers within the circumscribed study site, results of the study are generally representative of prevailing situations in these five Health Service Areas, which include $50 \%$ of the total hospital beds in Lesotho. Like all self-report surveys, our results are subject to accuracy and recall bias of the respondents. We sought to capture the prescribers' general attitudes and behavior rather than a snapshot. Further studies might refine our estimates by comparing responses to patient case files.

\section{Implications of the results for policy, clinical practice or further research}

Our results highlight healthcare provider related factors that contribute to inappropriate antibiotic prescribing in Lesotho. Considering the large number of responses "sometimes" or "at times" to most survey items, there is potential for correct prescribing, though in practice, the providers encounter difficulties.

We establish that healthcare providers tend to not use laboratory derived information in infection diagnosis and antibiotic treatments, which contributes to inappropriate antibiotic prescribing. We provide 
baseline information for policy formulation in addressing care providers' approach to antibiotic prescribing in clinical practice.

\section{Conclusion}

We show that healthcare providers in Lesotho public health institutions tend to prescribe antibiotics without adequately establishing the presence and characteristics of bacterial pathogens as aetiologies of treated clinical cases. Delays in receipt of laboratory results and the providers' high patient workload contribute to the under-utilization of laboratory facilities. Providers similarly reported prescribing antibiotics at times in cases of unsure diagnosis. Patients' request or demand for antibiotics and their expectations appear to influence nurses but not doctors. Fortunately, nearly all providers recognize that guidelines and refresher courses would be useful, similar to previous reports by physicians in Ghana ${ }^{18}$, in Scotland and France ${ }^{19}$, and in Chicago, $\mathrm{USA}^{20}$. These findings highlight the need for prescriber education on infection diagnosis and principles of antibiotic prescribing in order to reverse the practice of inadvertent antibiotic prescribing for purposes other than their indicated uses.

\section{Acknowledgements}

We acknowledge and are grateful for the part funding of this research by the National University of Lesotho.

\section{References}

1. World Health Organisation. WHO global strategy for containment of antimicrobial resistance. Geneva: World Health Organization. 2001.

2. Flie TM, Hadley JA. Rational use of antibiotics to treat respiratory tract infections. Am J Manag Care. 2002; 8: 713-27.

3. Bisht R, Katiyar A, Singh R. Mittal P. Antibiotic resistance - A global issue of concern. Asian J Pharma Clin Res. 2009; 2(2): 34 - 39.

4. Alanis AJ. Resistance to antibiotics: are we in the post-antibiotic era? Arch Med Res. 2005; 36: 697 705.

5. Levy SB. The Antibiotic Paradox: How the Misuse of Antibiotics Destroys Their Curative Powers, 2nd ed. Cambridge, MA: Perseus Publishing; 2002.

6. Crowther-Gibson P, Govender N, Lewis DA, Bamford C, Brink A, von Gottberg A, et al. Part IV: Human infections and antibiotic resistance. S Afr Med J. 101 (8): 567-578.
7. MacDougall C, Polk RE. Antimicrobial Stewardship Programs in Health Care Systems. Clin Microbiol Rev. 2005; 18(4):638-656.

8. Fishman N. Antimicrobial Stewardship. American Journal of Infection Control. 2006; 34 (5) ; S55-63.

9. Mangione-Smith R, Mcglyn EA, Elliot MN, Krogstad P, Brook RH. The relationship between perceived parental expectations and paediatrician antibiotic prescribing. Pediatrics. 1999; 103: 71118.

10. Gaur AH, English BK. The judicious use of antibiotics - An investment towards optimised health care. Indian J Paediatr. 2006; 73(4): 34350.

11. Gonzales R, Bartlett JG, Besser RE, Hickner JM, Hoffman JR, Sande MA. Principles of appropriate antibiotic use for treatment of nonspecific upper respiratory tract infections in adults: background. Ann Intern Med. 2001; 134(6): 490-4.

12. Archibald LK, Reiller LB. Clinical microbiology in developing countries. Emerg Infect Dis. 2001; 7(2): 302-304. 08;-1-24. 10

13. Aldridge A, Levine K. Surveying the social world: principles and practice in survey research. Buckingham: Open University Press. 2001.

14. Cho HJ, Hong SJ, Park S. Knowledge and beliefs of primary care physicians pharmacists and parents on antibiotic use for the pediatric common cold. Soc Sci Med. 2004; 58 (3): 623 629.

15. Radyowijati A, Haak H. Improving antibiotic use in low income countries: an overview of evidence on determinants. Soc Sci Med. 2003; 57(4): 733-44.

16. Miller E, MacKeigan LD, Rosser W, Marshman J. Effects of perceived patient demand on prescribing anti-infective drugs. Can Med Assoc J. 1999; 161: 139-42.

17. Palikhe N. Prescribing pattern of antibiotics in paediatric hospital of Kathmandu valley. Kathmandu Univ Med J. 2004; 2(1): 6-12.

18. Bosu WK, Ofori-Adjei D. Survey of antibiotic prescribing pattern in government health facilities of the Wassa west district of Ghana. East Afr Med J. 1997 Mar;74(3):138-42.

19. Pulcini C, Williams F, Molinari N, Davey P, Nathwani D. Junior doctors' knowledge and perceptions of antibiotic resistance and prescribing: a survey in France and Scotland. Clin Microbiol Infect 2011; 17: 80-87.

20. Wester CM, Durairaj L, Evans AT, Schwartz DN, Husain S, Martinez E. Antibiotic Resistance: A survey of physician perceptions. Arch Intern Med. 2002;162(19):2210-2216. 